UMD-PP-00-82

UW/PT-00-14

\title{
Radion Mediated Supersymmetry Breaking
}

\author{
Z. Chacko \\ Department of Physics, Box 351560 \\ University of Washington \\ Seattle, Washington 98195 \\ zchacko@fermi . phys. washington. edu \\ Markus A. Luty \\ Department of Physics \\ University of Maryland \\ College Park, Maryland 20742 \\ mluty@physics . umd .edu
}

\begin{abstract}
We point out that in supersymmetric theories with extra dimensions, radius stabilization can give rise to a VEV for the $F$ component of the radius modulus. This gives an important contribution to supersymmetry breaking of fields that propagate in the bulk. A particularly attractive class of models is obtained if the standard-model gauge fields propagate in the bulk, while the quark and lepton fields are localized on a brane. This leads to gaugino mediated supersymmetry breaking without the need for singlets in the hidden sector. We analyze a simple explicit model in which this idea is realized.
\end{abstract}

August 9, 2000 
Supergravity (SUGRA) mediated supersymmetry (SUSY) breaking is arguably the simplest and most natural mechanism for realizing SUSY in nature [1]. Because SUGRA couples to everything, it necessarily connects the observable and hidden sectors, and is therefore a natural candidate for communicating SUSY breaking to the observable sector. The main challenges for this class of models are explaining the absence of flavor-changing squark and slepton mass terms, the absence of $\mathrm{CP}$ violation in soft parameters, and the origin of the $\mu$ term.

The minimal version of SUGRA mediation assumes that all higher-dimension operators that connect the hidden and observable sectors are present and suppressed only by powers of $1 / M_{4}$, where $M_{4}$ is the 4 -dimensional Planck scale. This gives all required soft SUSY breaking terms (including the $\mu$ term [2]) of order $m_{3 / 2}$ from higher-dimension operators of the form

$$
\begin{aligned}
\mathcal{L}_{\mathrm{eff}} \sim \int d^{4} \theta \frac{1}{M_{4}^{2}} X^{\dagger} X Q^{\dagger} Q+\int d^{2} \theta \frac{1}{M_{4}} X \operatorname{tr} W^{\alpha} W_{\alpha}+\text { h.c. } \\
+\int d^{4} \theta\left(\frac{1}{M_{4}} X^{\dagger} H_{u} H_{d}+\frac{1}{M_{4}^{2}} X^{\dagger} X H_{u} H_{d}+\text { h.c. }\right)
\end{aligned}
$$

Here, $X$ is a chiral superfield in the hidden sector whose $F$ component breaks SUSY, $Q$ is a visible sector matter field, $H_{u}$ and $H_{d}$ are Higgs fields, and $W_{\alpha}$ is the field strength of the standard model gauge fields. Note that the terms that generate the gaugino masses and $\mu$ term require that $X$ be a singlet. This scenario is very simple, but it gives no explanation of the absence of flavor changing scalar masses or $\mathrm{CP}$ violation.

A very interesting variation on this scenario is anomaly-mediated SUSY breaking (AMSB) [3, 4. In this scenario, one assumes that the contact terms in Eq. (1) connecting the hidden and observable sector are suppressed. This occurs naturally if the hidden and observable sectors are localized on different 'branes' in a higherdimensional theory [3]. The superconformal anomaly then generates soft masses and $A$ terms of order $m_{3 / 2} / 16 \pi^{2}$ [3, 团. The minimal version of AMSB predicts negative slepton masses, but non-minimal visible sectors can make the scenario realistic [5, 6, 7]. Perhaps the most attractive models are those based on the observation of Ref. [5], that thresholds determined by moduli fields can change the AMSB predictions and make them realistic. An intriguing possibility is that the GUT threshold plays an important role [8]. The SUSY breaking parameters are naturally real (except possibly the $\mu$ term), which is sufficient to suppress all CP violation except for the QCD vacuum angle. The $\mu$ term requires a separate mechanism; it can be generated in a relatively simple way as the $\mathrm{VEV}$ of a singlet [5, 6]. 
Perhaps the simplest version of supergravity mediation is gaugino mediated SUSY breaking ( $\tilde{\mathrm{g} M S B}$ ) [9, 10]. This scenario is obtained if the standard-model (or GUT) gauge multiplets propagate in the bulk of a higher-dimension theory, while the quark and lepton fields are localized on the visible sector brane. If the hidden sector is localized on a different brane, the gauginos can obtain direct contributions to their mass from contact interactions with the hidden sector of the form (in 5 dimensions)

$$
\Delta \mathcal{L}_{5} \sim \delta(y) \int d^{4} \theta \frac{1}{M_{5}} X \operatorname{tr} W^{\alpha} W_{\alpha}+\text { h.c. },
$$

similar to minimal SUGRA models. The scalar masses are generated by 1-loop diagrams involving the gauge fields. The scalar masses are therefore loop suppressed at the compactification scale, but RG evolution between the compactification scale and the weak scale can give rise to a realistic superpartner spectrum. The resulting phenomenology is similar to 'no-scale' supergravity models [1]1], but it is important to take into account the running above the GUT scale [12, 13] and/or possible FayetIliopoulos terms [10]. Also, it is important that the geometrical set-up of g MSB gives an understanding of the 'no-scale' structure, and why it is stable under radiative corrections. This scenario naturally solves the SUSY flavor problem because the gauge couplings that communicate SUSY breaking to the squarks and sleptons are flavorblind. It also solves the SUSY CP problem because the SUSY breaking sector is localized on a different brane where CP can be a good symmetry. On the other hand, gMSB by itself does not explain the value of the $\mu$ term.

In $\tilde{g} \mathrm{MSB}$, the gaugino masses are conventionally assumed to arise from a contact terms of the form Eq. (2). This however requires that the field $X$ that breaks supersymmetry be a singlet. It is interesting to ask what is the leading SUSY breaking effect in the visible sector if the standard model gauge fields are in the bulk but the hidden sector contains no singlets with large $F$ terms. A reasonable guess might be anomaly mediation, since the largest competing contact interaction

$$
\Delta \mathcal{L}_{5} \sim \delta(y) \int d^{4} \theta \frac{1}{M_{5}^{2}} X^{\dagger} X\left(\operatorname{tr} W^{\alpha} W_{\alpha}+\text { h.c. }\right)
$$

is much smaller. However as we shall show this need not be the case. There is a large class of theories in which the volume of the extra dimensions is undetermined in the SUSY limit, and corresponds to a flat direction called the radius modulus or 'radion.' The low-energy theory below the compactification scale will then contain a radion chiral superfield $T$. In order for such a scenario to be realistic, the radius must be

\footnotetext{
${ }^{1}$ In some versions of this model, the Higgs fields propagate in the bulk, and therefore obtain soft scalar masses, $\mu$, and $B \mu$ terms directly from contact interactions with the hidden sector [10].
} 
stabilized when SUSY is broken, which corresponds to the radion acquiring a mass. In this paper, we point out that stabilizing the radius can generate a $\mathrm{VEV}$ for $F_{T}$. This gives rise to direct (tree level) SUSY breaking terms for fields that propagate in the bulk. Similar effects are well-known in string phenomenology 14. If the gauginos propagate in the bulk, this naturally gives rise to a version of gaugino mediation that we call 'radion mediated SUSY breaking' (RMSB). We will analyze an explicit model in which the radion is stabilized, which realizes these ideas.

We work for concreteness in a model with 5 spacetime dimensions, with one dimension compactified on a $S^{1} / Z_{2}$ orbifold with radius $r$. The orbifold projection breaks the $\mathcal{N}=2$ SUSY in 5 dimensions down to $\mathcal{N}=1$ in 4 dimensions, and 3branes may be naturally fixed at the orbifold fixed points. We assume for simplicity that the 5-dimensional spacetime is approximately flat, although the generalization of these results to strongly 'warped' spacetimes [15] is interesting. In SUSY theories, the radion is part of a chiral superfield modulus $T$, with

$$
\operatorname{Re}(T) \propto r, \quad \operatorname{Im}(T) \propto B_{5},
$$

where $B_{5}$ is the $5^{\text {th }}$ component of the graviphoton in 5-dimensional SUGRA. 20 Stabilizing the radius requires additional bulk dynamics that is sensitive to $r$. As we will see in an explicit model, this can give rise to a VEV for $F_{T}$, breaking SUSY.

We now work out the effects of $\left\langle F_{T}\right\rangle$ on a bulk gauge field. In the 4-dimensional effective lagrangian, the gauge coupling of the gauge zero mode is given by

$$
\frac{1}{g_{4}^{2}}=\frac{2 \pi r}{g_{5}^{2}}
$$

where $g_{5}$ is the 5 -dimensional gauge coupling (with mass dimension $-\frac{1}{2}$ ). To generalize this relation to superspace, note that the kinetic term for the gauge zero mode is

$$
\Delta \mathcal{L}_{4}=\int d^{2} \theta S_{4} \operatorname{tr}\left(W^{\alpha} W_{\alpha}\right)+\text { h.c. }
$$

where $S_{4}$ is the holomorphic gauge coupling. The unique SUSY generalization of Eq. (5) is

$$
S_{4} \propto \frac{T}{g_{5}^{2}}
$$

From Eqs. (6) and (7) we obtain a nonzero gaugino mass

$$
m_{\lambda}=\frac{\left\langle F_{T}\right\rangle}{2\langle T\rangle} .
$$

\footnotetext{
${ }^{2}$ With this choice, the 4-dimensional SUGRA multiplet and $T$ to transform independently under $\mathcal{N}=1$ SUSY. For an effective field theory derivation of this fact (and Eq. (雨), see Ref. [16.
} 
We will not consider the analogous results for a bulk hypermultiplet here. We cannot forbid contact terms between bulk hypermultiplets and the hidden sector, and therefore the SUSY breaking terms are not completely determined by $\left\langle F_{T}\right\rangle /\langle T\rangle$ for bulk hypermultiplets.

To know whether the contribution Eq. (8) dominates over other contributions (such as anomaly mediation), we need to estimate the quantity $\left\langle F_{T}\right\rangle /\langle T\rangle$. This requires a radius stabilization mechanism. We will consider the model described in Ref. [16. The additional ingredients required to stabilize the radius are a $S U(N)$ super Yang-Mills (SYM) sector in the bulk and a $S U(\tilde{N})$ SYM sector on one of the branes. Below the compactification scale, the bulk SYM theory becomes a 4dimensional SYM sector with a gauge coupling that depends on the radion as in Eq. (7). Both SYM sectors give rise to gaugino condensation in the 4-dimensional theory, generating a dynamical superpotential

$$
W_{\text {eff }}=a e^{-b T}+c .
$$

The first term arises from gaugino condensation in the bulk SYM sector, and the second term in the superpotential arises from the SYM sector on the brane. Normalizing $\operatorname{Re}(T)=3 \pi r$ as in Ref. [16], we have

$$
b=\frac{32 \pi^{2}}{3 g_{5}^{2} N} .
$$

Using 'naïve dimensional analysis' [17] we estimate

$$
a \sim \frac{\Lambda_{\mathrm{UV}}^{3}}{16 \pi^{2} N}, \quad c \sim \frac{\Lambda_{\mathrm{IR}}^{3}}{16 \pi^{2} \tilde{N}}
$$

where $\Lambda_{\mathrm{IR}}$ is the scale where the brane SYM sector becomes strong in the IR, and $\Lambda_{\mathrm{UV}}$ is the scale where the 5-dimensional bulk SYM sector becomes strong in the UV.

In addition to the radius stabilization sector described above, it is assumed that SUSY is broken on the hidden sector brane.

To describe the complete effective lagrangian, we use the superconformal formulation of 4-dimensional $\mathcal{N}=1$ SUGRA 18 . For our results, it will be sufficient to know the couplings of the scalar auxiliary field of the SUGRA multiplet. These can be parameterized by the conformal compensator $\phi$, a chiral superfield with components

$$
\phi=1+\theta^{2} F_{\phi}
$$

The couplings of $\phi$ are completely determined by a spurious $U(1)_{R}$ and dilatation symmetry under which $R(\phi)=\frac{2}{3}, d(\phi)=1$. In a basis where all matter and gauge 
fields have vanishing $U(1)_{R}$ charge and dilatation weight, the lagrangian can be written

$$
\mathcal{L}_{4}=\int d^{4} \theta \phi^{\dagger} \phi f+\left(\int d^{2} \theta \phi^{3} W+\text { h.c. }\right)
$$

where $W$ is the superpotential, and $f$ is related to the canonically defined Kähler potential $K$ by $f=-3 e^{-K / 3 M_{4}^{2}}$. Cancelling the cosmological constant after SUSY breaking gives $\left\langle F_{\phi}\right\rangle \sim m_{3 / 2}$. In this notation, the 4-dimensional effective theory for the model described above is

$$
\begin{aligned}
\mathcal{L}_{4}=\int & d^{4} \theta \phi^{\dagger} \phi\left[-M_{5}^{3}\left(T+T^{\dagger}\right)+f_{\text {vis }}+f_{\text {hid }}\right] \\
+ & {\left[\int d^{2} \theta \phi^{3}\left(a e^{-b T}+c+W_{\text {vis }}+W_{\text {hid }}\right)+\text { h.c. }\right]+\mathcal{O}\left(1 / M_{4}^{4}\right) } \\
& -V_{\text {hid }} \times[1+\text { Goldstino terms }] .
\end{aligned}
$$

The Kähler potential has the standard 'no scale' form. $V_{\text {hid }}$ is the vacuum energy from SUSY breaking in the hidden sector. Below the SUSY breaking scale, SUSY will be nonlinearly realized with additional Goldstino terms that are not relevant here. We will assume that $a$ and $c$ are real, which corresponds to a discrete choice of vacua for the gaugino condensates. Minimizing the resulting potential, we find solutions with $\langle T\rangle$ real and $b\langle T\rangle \gg 1$ for $c \ll a$. We also obtain

$$
\frac{\left\langle F_{T}\right\rangle}{\langle T\rangle}=\frac{2}{b\langle T\rangle}\left\langle F_{\phi}\right\rangle,
$$

which gives SUSY breaking suppressed by a volume factor compared to $\left.\left\langle F_{\phi}\right\rangle \cdot\right]^{3}$ If the standard model gauge multiplet propagates in the bulk, this gives a contribution to the gaugino masses via Eq. (8).

The radion mediated contribution to the gaugino mass from Eq. (15) will be large than the anomaly mediated contribution provided that $b\langle T\rangle$ is not too large. To obtain numerical estimates, we consider the case where the bulk SYM sector and gravity become strong at the same scale, which gives $M_{5}^{3} \sim \Lambda_{\mathrm{UV}}^{3} / 128 \pi^{3}$. We then solve for $b\langle T\rangle$ using

$$
\frac{\left\langle F_{\phi}\right\rangle}{M_{4}}=\frac{a}{M_{4}^{3}} b\langle T\rangle e^{-b\langle T\rangle} .
$$

From $\left\langle F_{\phi}\right\rangle \sim m_{3 / 2} \sim 10-100 \mathrm{TeV}$, we obtain $b\langle T\rangle \simeq 32$. This estimate is dominated by the exponent in Eq. (16), and is therefore insensitive to the estimates used above.

\footnotetext{
${ }^{3}$ This suppression was missed in Ref. [16], but does not affect the conclusions of that paper.
} 
We then obtain

$$
\frac{\left\langle F_{T}\right\rangle}{\langle T\rangle} \sim \frac{1}{16}\left\langle F_{\phi}\right\rangle, \quad r \Lambda_{\mathrm{UV}} \sim 25
$$

Note that the radius is sufficiently small that the standard-model gauge coupling is perturbative up to the scale $\Lambda_{\mathrm{UV}}$. On the other hand, the radius is large enough to suppress the exchange of massive states (e.g. excited string modes or extended objects) between the hidden and visible sector branes. These are potentially dangerous because they can give rise to contact terms of the form

$$
\Delta \mathcal{L}_{4} \sim \int d^{4} \theta \frac{e^{-M r}}{M^{2}} X^{\dagger} X Q^{\dagger} Q
$$

where $M$ is the mass of the heavy states. Since we expect $M \sim \Lambda_{\mathrm{UV}}$, this is sufficient to suppress FCNC's.

We see that in this model the radion-mediated contribution to the gaugino mass is about an order of magnitude smaller than $m_{3 / 2}$. However, it is still larger than the anomaly mediated contribution $\Delta m_{\lambda} \sim m_{3 / 2} / 16 \pi^{2}$. If the compactification scale is above the GUT scale, it may appear that large multiplicity factors in loops above the GUT scale can make the anomaly-mediated contribution to the gaugino mass comparable to (or larger than) the radion-mediated contribution. However, this generally does not happen. Note that at the compactification scale, the gaugino mass is (up to loop matching corrections) the sum of the AMSB and RMSB contributions. (Cross terms arising from e.g. radion dependent SUSY breaking in the regulators is loop suppressed compared to the direct RMSB contribution.) RG evolution from the compactification scale down to the GUT scale corrects this by factors that are loop suppressed compared to the gaugino mass at the compactification scale. Even if the AMSB contribution dominates above the GUT scale, the RG is dominated by the AMSB trajectory, and the the gaugino mass at the GUT scale is dominantly AMSB, with an additive correction equal to the RMSB contribution. If the GUT scale is a SUSY threshold (unlike the model of Ref. [8]) there are large loop matching contributions that will reduce the AMSB contributions to the corresponding MSSM values below the GUT scale. One is therefore left with the RMSB contribution with a small AMSB correction. A brief calculation shows that just below the GUT scale the ratio of the magnitudes of the two contributions is approximately 3:1 for the gluino, 10:1 for the wino and 3:2 for the bino, and the total gaugino mass is the sum (or difference) of the two contributions. To avoid a small bino mass, we require that the AMSB and RMSB contributions have the same sign, which is obtained for an appropriate choice of sign for $a$ in the model above. The sizable correction to the bino mass in this model will show up as a correction to gaugino unification in this model. 
A similar argument shows that the AMSB contributions to the scalar masses are small below the GUT scale.

We conclude that gaugino mediation arises naturally in this model. The corrections to the bino mass will increase the slepton masses, which may have important phenomenological implications. We will leave this for future work.

In conclusion, we have shown that that radius stabilization can give rise to a VEV for the $F$ component of the radion modulus, which can give an important contribution to SUSY breaking of bulk fields. If the standard model (or GUT) gauginos propagate in the bulk, while the other fields are localized on branes, we naturally obtain gaugino mediated SUSY breaking without the need for singlets in the hidden sector. We have analyzed a specific radius stabilization mechanism to illustrate these ideas and give a completely realistic model. We hope that these ideas will show the way to even simpler realistic models, perhaps even in the context of string theory.

Note added: While this paper was being completed, we received Ref. [19, which considers related ideas.

\section{Acknowledgments}

We thank R. Sundrum for discussions. Z.C. was supported by the DOE under contract DE-FG03-96-ER40956. M.A.L. was supported by the NSF under grant PHY-9802551.

\section{References}

[1] A.H. Chamseddine, R. Arnowitt, P. Nath, Phys. Rev. Lett. 49, 970 (1982); R. Barbieri, S. Ferrara, C.A. Savoy, Phys. Lett. 119B, 343 (1982); L.J. Hall, J. Lykken, S. Weinberg, Phys. Rev. D27, 2359 (1983). For a review, see H.P. Nilles, Phys. Rep. 110, 1 (1984).

[2] G.F. Giudice, A. Masiero, Phys. Lett. 206B, 480 (1988).

[3] L. Randall and R. Sundrum, hep-th/9810155.

[4] G.F. Giudice, M.A. Luty, H. Murayama, R. Rattazzi, hep-ph/9810442.

[5] A. Pomarol, R. Rattazzi, JHEP 9905:013 (1999), hep-ph/9903448.

[6] Z. Chacko, M.A. Luty, I. Maksymyk, E. Pontón, JHEP 0004:001 (2000), hep-ph/9905390. 
[7] E. Katz, Y. Shadmi, Y. Shirman, JHEP 9908:015 (1999), hep-ph/9906296.

[8] Z. Chacko, M.A. Luty, E. Pontón, Y. Shadmi, Y. Shirman, hep-ph/0006047.

[9] D.E. Kaplan, G.D. Kribs, M. Schmaltz, hep-ph/9911293.

[10] Z. Chacko, M.A. Luty, A.E. Nelson, E. Pontón, JHEP 0001:003 (2000), hep-ph/9911323.

[11] J. Ellis, K. Enqvist, D.V. Nanopoulos, Phys. Lett. 147B, 99 (1984); J. Ellis, C. Kounnas, D.V. Nanopoulos, Nucl. Phys. B247, 373 (1984); For a review, see A.B. Lahanas, D.V. Nanopoulos, Phys. Rep. 145, 1 (1987).

[12] M. Schmaltz, W. Skiba, hep-ph/0001172.

[13] M. Schmaltz, W. Skiba, hep-ph/0004210.

[14] See e.g. B. de Carlos, J.A. Casas, C. Muñoz, Nucl. Phys. B399, 623 (1993); V. Kaplanuvsky, J. Louis, Phys. Lett. 306B, 269 (1993); A. Brignole, L.E. Ibañez, C. Muñoz, Nucl. Phys. B422, 125 (1994) [Erratum: Nucl. Phys. B436, 747 (1995)].

[15] L. Randall, R. Sundrum, Phys. Rev. Lett. 83, 3370 (1999), hep-ph/9905221.

[16] M.A. Luty, R. Sundrum, hep-ph/9910202, to appear in Phys. Rev. D.

[17] A.V. Manohar, H. Georgi, Nucl. Phys. B234, 189 (1984); M.A. Luty, Phys. Rev. D57, 1531 (98), hep-ph/9706235; A.G. Cohen, D.B. Kaplan, A.E. Nelson, Phys. Lett. 412B, 301 (97), hep-ph/9706275; Z. Chacko, M.A. Luty, E. Pontón, hep-ph/9909248, to appear in JHEP.

[18] K.S. Stelle, P.C. West, Phys. Lett. 74B, 330 (1978); S. Ferrara, P. van Nieuwenhuizen, Phys. Lett. 74B, 333 (1978); E. Cremmer, S. Ferrara, L. Girardello, A. Van Proeyen, Nucl. Phys. B212, 413 (1983).

[19] T. Kobayashi, K. Yoshioka, hep-ph/0008069. 